\title{
A NOTE ON A CONNECTION BETWEEN THE POINCARÉ-ARNOLD-MELNIKOV INTEGRAL AND THE PICARD-VESSIOT THEORY
}

\author{
JUAN J. MORALES-RUIZ \\ Departament de Matemàtica Aplicada II, Universitat Politècnica de Catalunya \\ Pau Gargallo 5, E-08028 Barcelona, Spain \\ E-mail:morales@ma2.upc.es
}

\begin{abstract}
We obtain an algebraic interpretation by means of the Picard-Vessiot theory of a result by Ziglin about the self-intersection of complex separatrices of time-periodically perturbed one-degree of freedom complex analytical Hamiltonian systems.

1. Introduction. Let there be given a one-degree of freedom real-analytic Hamiltonian system $X_{H_{0}}$ with Hamiltonian $H_{0}(x, y)$, a hyperbolic equilibrium point and a particular integral curve $\Gamma_{0}:\left(x_{0}(t), y_{0}(t)\right)$ asymptotic to the equilibrium point. For instance, $\Gamma_{0}$ can be a homoclinic integral curve. The problem considered is to study the integrability of this system under small periodic perturbations. More concretely, we consider a real-analytic Hamiltonian $H(x, y, t, \varepsilon)=H_{0}(x, y)+\varepsilon H_{1}(x, y, t)+$ h.o.t. $(\varepsilon)$, where $\varepsilon$ is a small parameter and $H$ depends periodically on $t$. A very effective tool to study the integrability of the perturbed system for sufficiently small $\varepsilon$ is the so-called Poincaré-Arnold-Melnikov integral ([14, 1, 11])

$$
\mu\left(t_{0}\right):=\int_{-\infty}^{\infty}\left\{H_{0}, H_{1}\right\}\left(x_{0}(t), y_{0}(t), t+t_{0}\right) d t,
$$

where $\{$,$\} denotes the Poisson bracket, i.e., \{f, g\}=\frac{\partial f}{\partial x} \frac{\partial g}{\partial y}-\frac{\partial f}{\partial y} \frac{\partial g}{\partial x}$. When the function $\mu\left(t_{0}\right)$ has simple zeros, the stable and unstable separatrices of the perturbed Hamiltonian system intersect transversally and this is an obstruction to the complete integrability of the system by means of analytical first integrals.

Now we consider the extension of system $X_{H}$ to the complex domain by extending all the variables (including time) to the complex plane. It was observed that there are some

2000 Mathematics Subject Classification: Primary 37J30; Secondary 34M35, 12H05, 34M15, $70 \mathrm{G} 65$.

This work was partially supported by the Spanish grant DGICYT PFM2000-0805 and the Catalan grant CIRIT 2000SGR-00027.

The paper is in final form and no version of it will be published elsewhere.
\end{abstract}


relation between the Poincaré-Arnold-Melnikov method and the branching of the solution of the perturbed system in complex time $[15,6]$. In particular, Ziglin roughly proved that, for perturbations of a 1-degree of freedom Hamiltonian systems, if the PoincaréArnold-Melnikov integral computed along some closed loop in the complex time plane has simple zeros, then the separatrix self-intersects transversally (see next section for a precise statement). The objective of this note is to give an algebraic interpretation of this result in the context of the Picard-Vessiot theory of linear differential equations. We essentially prove that, under some assumptions, the above condition about the PoincaréArnold-Melnikov integral given by Ziglin can be interpreted by the fact that the Galois group of the perturbed variational equation along the integral curve $\Gamma_{0}$ is non-abelian. This result is similar to a joint result of the author with Ramis [13] stating that a necessary condition for integrability is that the identity component of the Galois group of the variational equation (along a particular integral curve) must be abelian (see section 5 below).

2. Ziglin's theorem about the splitting of separatrices. Let $M_{0}$ be a twodimensional complex analytic symplectic manifold and $X_{H_{0}}$ a complex holomorphic Hamiltonian field on $M_{0}$ with Hamiltonian $H_{0}$. Locally, in symplectic coordinates, $H_{0}$ is a function of $x, y \in \mathbf{C}^{2}$. Assume that the corresponding Hamiltonian system

$$
\dot{x}=\frac{\partial H_{0}}{\partial y}, \quad \dot{y}=-\frac{\partial H_{0}}{\partial x}
$$

has a hyperbolic equilibrium point $\left(x_{0}, y_{0}\right)$, i.e., the eigenvalues $\pm \lambda$ of the linear part of $X_{H_{0}}$ at $\left(x_{0}, y_{0}\right)$ have non-zero real parts. Then system (1) has a separatrix

$$
\Gamma_{0}:\left(x_{0}(t), y_{0}(t)\right), \quad \lim _{t \rightarrow \infty} x_{0}(t)=x_{0}, \quad \lim _{t \rightarrow \infty} y_{0}(t)=y_{0} .
$$

We assume that the functions $x_{0}(t), y_{0}(t)$ are meromorphic in $t \in \mathbf{C}$. Let

$$
H(x, y, t, \varepsilon)=H_{0}(x, y)+\varepsilon H_{1}(x, y, t)+\text { h.o.t. }(\varepsilon)
$$

be a meromorphic small (complex) perturbation of $H_{0}$. Furthermore we assume that $H$ is periodic in $t$ with real period $2 \pi$. This function $H$ is defined over $M=M_{0} \times F_{2 \pi}$, where $F_{2 \pi}=\mathbf{C} / 2 \pi \mathbf{Z}$ is the Riemann surface defined by the period. In general, we write $F_{\omega}=\mathbf{C} / \omega \mathbf{Z}$ for the Riemann surface defined by a period $\omega \in \mathbf{C}$. The Hamiltonian system defined by $H=H(x, y, \theta)$ over $M$ is given by

$$
\dot{x}=\frac{\partial H}{\partial y}, \quad \dot{y}=\frac{-\partial H}{\partial x}, \quad \dot{\theta}=1, \quad(x, y, \theta) \in M .
$$

It is convenient to consider the unperturbed system (1) as defined over $M$, i.e., given by the direct product of (1) and $\dot{\theta}=1$ :

$$
\dot{x}=\frac{\partial H_{0}}{\partial y}, \quad \dot{y}=\frac{-\partial H_{0}}{\partial x}, \quad \dot{\theta}=1, \quad(x, y, \theta) \in M .
$$

For $\varepsilon=0$, system (3) reduces to system (4) and it has a hyperbolic $2 \pi$-periodic orbit $\Pi_{0}:\left(x_{0}, y_{0}, \theta=t(\bmod 2 \pi)\right)$. It is well-known that, for small $|\varepsilon|$, system $(3)$ has also a $2 \pi$ periodic solution $\Pi_{\varepsilon}:\left(x_{p}(t, \varepsilon), y_{p}(t, \varepsilon), \theta=\theta_{0}+t(\bmod 2 \pi)\right)$, such that $\left(x_{p}(t, 0), y_{p}(t, 0)\right)=$ $\left(x_{0}, y_{0}\right)$. Then we define the (stable) complex separatrix $\Lambda_{\varepsilon}^{+}$of system (3) as the set of 
integral curves of (3) asymptotic to $\Pi_{\varepsilon}$ as $t \rightarrow \infty$. For fixed $\varepsilon$, it is a two-dimensional complex surface. This separatrix can have transverse self-intersections.

We recall that in the real case the separatrices can not have transverse self-intersections, transverse intersections can only occur between the stable and unstable separatrices. For real Hamiltonian systems, the existence of such transverse orbits is one of the typical ingredients of chaotic behavior and is an obstruction to the existence of an additional analytical first integral. Ziglin proved that, for complex Hamiltonian systems, the existence of transverse self-intersections for separatrices is also an obstruction to the integrability of system (3); a sufficient condition for this behavior is given by the Poincaré-Arnold-Melnikov integral computed along closed paths.

The unperturbed separatrix is given by $\Lambda_{0}^{+}=\Gamma_{0} \times F_{2 \pi}$. It is foliated by the 1parameter family of integral curves

$$
\Gamma_{\theta_{0}}:\left(x_{0}(t), y_{0}(t), t+\theta_{0}\right),
$$

$\theta_{0} \in F_{2 \pi}$ (the parameter is $\left.\theta_{0}\right)$.

Let $\gamma:[0,1] \rightarrow \mathbf{C}$ be a closed path in the complex plane, with $\gamma(0)=\gamma(1) \in \mathbf{R} \subset \mathbf{C}$. We define the function on $F_{2 \pi}$

$$
\mu\left(\theta_{0}\right):=\int_{\gamma}\left\{H_{0}, H_{1}\right\}\left(x_{0}(t), y_{0}(t), t+\theta_{0}\right) d t .
$$

This is the Poincaré-Arnold-Melnikov integral in this context. Then

THEOREM 1 ([15]). If the function $\mu\left(\theta_{0}\right)$ has a simple zero, then for sufficiently small $|\varepsilon| \neq 0$, the separatrix $\Lambda_{\varepsilon}^{+}$has a transversal self-intersection and system (3) has no additional holomorphic first integral.

If we consider system (3) as a family of two-degrees of freedom Hamiltonian systems (the conjugated canonical variable to $t$ is the energy), then the conclusion of Ziglin's theorem is that, for $|\varepsilon| \neq 0$ small, these systems are not completely integrable with holomorphic first integrals.

3. Galois differential approach. We assume that the reader is familiar with the main facts about the Picard-Vessiot theory of linear differential equations $[5,8,9,10]$.

Along the rest of this note we assume that the meromorphic functions $x_{0}(t), y_{0}(t)$ are periodic in the imaginary direction with period $i \omega$. Then $x_{0}(t), y_{0}(t) \in \mathcal{M}\left(F_{i \omega}\right)$ the field of meromorphic functions over the Riemann surface defined by the period $i \omega$. We remark that this assumption is always satisfied if $\Gamma_{0}$ is an homoclinic orbit. It is so because essentially, as $t \rightarrow \pm \infty$ along the real line, the dominant part of $x_{0}(t), y_{0}(t)$ is given by exponentials and the period is $\omega=\operatorname{Re}\left(\frac{2 \pi}{|\lambda|}\right)$, where $\pm \lambda$ are the eigenvalues of the hyperbolic equilibrium point $\left(x_{0}, y_{0}\right)$ (see [16] for details).

The Poincaré-Arnold-Melnikov method is usually based on the variation of constants formula applied to the perturbed variational equation, along the unperturbed 1-parameter family of integral curves $\Gamma_{\theta_{0}}$ (see [7]). We are going to study the differential Galois group of this equation. 
The perturbed variational equation $(P V E)$ of $(3)$ along $\Gamma_{\theta_{0}}$ is given by

$$
\frac{d}{d t}\left(\begin{array}{c}
\xi \\
\eta \\
\nu
\end{array}\right)=\left(\begin{array}{ccc}
H_{0 x y} & H_{0 y y} & H_{1 y} \\
-H_{0 x x} & -H_{0 x y} & -H_{1 x} \\
0 & 0 & 0
\end{array}\right)\left(\begin{array}{c}
\xi \\
\eta \\
\nu
\end{array}\right),
$$

where for simplicity we write $H_{0 x y}, H_{0 y y}, H_{0 x x}, H_{1 y}, H_{1 x}$ for the restrictions of these functions to $\Gamma_{\theta_{0}}$, i.e., $H_{0 x y}=H_{0 x y}\left(x_{0}(t), y_{0}(t)\right), H_{0 y y}=H_{0 y y}\left(x_{0}(t), y_{0}(t)\right), H_{0 x x}=$ $H_{0 x x}\left(x_{0}(t), y_{0}(t)\right), H_{1 y}=H_{1 y}\left(x_{0}(t), y_{0}(t), t\right), H_{1 x}=H_{1 x}\left(x_{0}(t), y_{0}(t), t+\theta_{0}\right)$.

We remark that equation $(7)$ is a homogeneous linear differential equation equivalent to the more classical $(P V E)$ written as an order-two linear inhomogeneous equation

$$
\frac{d}{d t}\left(\begin{array}{c}
\xi \\
\eta
\end{array}\right)=\left(\begin{array}{cc}
H_{0 x y} & H_{0 y y} \\
-H_{0 x x} & -H_{0 x y}
\end{array}\right)\left(\begin{array}{c}
\xi \\
\eta
\end{array}\right)+\left(\begin{array}{c}
H_{1 y} \\
-H_{1 x}
\end{array}\right),
$$

see $[7,1]$. The homogeneous part of $(8)$ is the variational equation of the integrable unperturbed system (1) along $\Gamma_{0}$.

A key point in any application of the Picard-Vessiot theory is the choice of the coefficient field $K$. In order to simplify this choice we assume that the functions $x_{0}(t), y_{0}(t)$ belong to the differential field $\mathbf{C}\left(e^{2 \pi t / \omega}\right)$ and also that the perturbation $H_{1}$ depends on $x, y$ as a rational function and on $t$ as a rational function in $e^{i t}$. In general, the above assumptions are sufficient for the applications. Under these assumptions we can take $K$ as $\mathbf{C}\left(e^{2 \pi t / \omega}, e^{i t}\right)$. Then it is clear that $K$ is a differential field and that the coefficients of equation (7) belong to this field.

For a given $\theta_{0}$, the Picard-Vessiot extension of (7) is given by $K \subset L_{\theta_{0}}:=K\left(u_{11}, \ldots\right.$ $\left.\ldots, u_{33}\right)$, where $U(t):=\left(u_{i j}(t)\right), i, j=1,2,3$, is a fundamental matrix of $(7)$. We write $\operatorname{Gal}(P V E)$ for the Galois group of this extension, i.e., $\operatorname{Gal}(P V E)=\operatorname{Gal}\left(L_{\theta_{0}} / K\right)$.

A fundamental matrix of (7) is given by

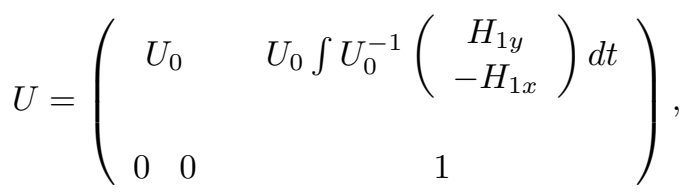

where $U_{0}$ is a fundamental matrix of the homogeneous part of equation (8)

$$
\frac{d}{d t}\left(\begin{array}{c}
\xi \\
\eta
\end{array}\right)=\left(\begin{array}{cc}
H_{0 x y} & H_{0 y y} \\
-H_{0 x x} & -H_{0 x y}
\end{array}\right)\left(\begin{array}{l}
\xi \\
\eta
\end{array}\right):=A\left(\begin{array}{c}
\xi \\
\eta
\end{array}\right) .
$$

The coefficients of this equation are in $\mathbf{C}\left(e^{2 \pi t / \omega}\right) \subset \mathbf{C}\left(e^{2 \pi t / \omega}, e^{i t}\right)=K$.

A fundamental matrix of (10) is

$$
U_{0}=\left(\begin{array}{cc}
H_{0 y} & a \\
-H_{0 x} & b
\end{array}\right),
$$

where $a$ and $b$ are given by quadratures of functions in $\mathbf{C}\left(e^{2 \pi t / \omega}\right)$. Indeed, equation (10) is the variational equation of the integrable system (1) along $\Gamma_{0}$. It is well-known that a particular solution of (10) is the Hamiltonian field along $\Gamma_{0},\left(H_{0 y},-H_{0 x}\right)$, then we can find the other independent solution by quadratures. Furthermore, the matrix $U_{0}$ can be 
chosen as a symplectic matrix, i.e., $\operatorname{det}\left(U_{0}\right)=1$, by the Hamiltonian structure of equation (10) (see $[13,12])$.

We remark that this is a very particular case of the process of reduction of the variational equation to the normal variational equation $[13,12]$. Let us make the computations explicit. We consider a canonical change of variables (change of the symplectic frame) $U_{0}=P V_{0}$, where $V_{0}$ is another fundamental system of (10)), with

$$
P:=\left(\begin{array}{cc}
H_{0 y} & 0 \\
-H_{0 x} & \frac{1}{H_{0 y}}
\end{array}\right) .
$$

We observe that $P$ is a symplectic matrix with coefficients in $K$; hence the new differential system will be equivalent to (10); in particular, their Picard-Vessiot extensions are the same and their Galois groups are isomorphic.

The new matrix of the system is given by

$$
P^{-1}(A P-\dot{P})=\left(\begin{array}{cc}
0 & \frac{H_{0} y y}{H_{0 y}^{2}} \\
0 & 0
\end{array}\right) \text {. }
$$

Then we obtain

$$
U_{0}=P V_{0}=\left(\begin{array}{cc}
H_{0 y} & 0 \\
-H_{0 x} & \frac{1}{H_{0 y}}
\end{array}\right)\left(\begin{array}{cc}
1 & \delta \\
0 & 1
\end{array}\right)=\left(\begin{array}{cc}
H_{0 y} & H_{0 y} \delta \\
-H_{0 x} & \frac{1}{H_{0 y}}-H_{0 x} \delta
\end{array}\right),
$$

where $\delta=\delta(t):=\int \frac{H_{0} y y}{H_{0 y}^{2}}\left(x_{0}(t), y_{0}(t)\right) d t$. As usual in differential algebra, we write $\int \frac{H_{0} y y}{H_{0 y}^{2}}\left(x_{0}(t), y_{0}(t)\right) d t$ for any primitive of the function $\frac{H_{0} y y}{H_{0 y}^{2}}\left(x_{0}(t), y_{0}(t)\right)$.

The Picard-Vessiot extension of (10) is given by a quadrature

$$
K \subset L_{1}=K(\delta) \text {. }
$$

We need two more assumptions. First, we assume that the general solution of equation (10) is uniform in $t$. By the above analysis this is equivalent to the uniformity of the function $\delta=\delta(t)$. That means that the residues of the function $\frac{H_{0} y y}{H_{0 y}^{2}}\left(x_{0}(t), y_{0}(t)\right.$ are zero. Generically this is always satisfied. For instance, in the case when the general solution of system (1) is uniform (because the solution of (10) are derivatives of solutions of (1)) or in the case when the Hamiltonian $H_{0}$ is a natural mechanical system $H_{0}=$ $\frac{1}{2} y^{2}+V(x)$ (because $\frac{H_{0} y y}{H_{0 y}^{2}}\left(x_{0}(t), y_{0}(t)\right)=\frac{1}{y_{0}^{2}}$ has poles of multiplicity at least two at points of zero velocity). We remark that in this case $L_{1} \subset \mathcal{M}(\mathbf{C})$, where $\mathcal{M}(\mathbf{C})$ is the field of meromorphic functions over $\mathbf{C}$.

Furthermore, we assume that $L_{1} \neq K$, i.e., $\delta \notin K$. As $\frac{H_{0} y y}{H_{0 y}^{2}}\left(x_{0}(t), y_{0}(t)\right) \in \mathbf{C}\left(e^{2 \pi t / \omega}\right)$, this condition can be checked by the fact that the integral along the period $i \omega$ must be non-zero. Then, by the standard exponential change, it turns into a non-zero residue of a rational function at the origin.

Under these assumptions we have that the Galois group of (10) is the additive group $\operatorname{Gal}\left(L_{1} / K\right)=G_{a}=(\mathbf{C},+)$. In the representation given by the fundamental matrix (12) we write it as

$$
\left\{\left(\begin{array}{cc}
1 & \lambda \\
0 & 1
\end{array}\right): \lambda \in \mathbf{C}\right\}
$$


Now we observe that (for a fixed $\theta_{0}$ ), using (11) and det $U_{0}=1$, the quadratures of the third column in the fundamental matrix (9) are given by

$$
\int U_{0}^{-1}\left(\begin{array}{c}
H_{1 y} \\
-H_{1 x}
\end{array}\right) d t=\left(\begin{array}{c}
\int\left(\frac{H_{1 y}}{H_{0 y}}-\delta\left\{H_{0}, H_{1}\right\}\right)\left(x_{0}(t), y_{0}(t), t+\theta_{0}\right) d t \\
\int\left\{H_{0}, H_{1}\right\}\left(x_{0}(t), y_{0}(t), t+\theta_{0}\right) d t
\end{array}\right) .
$$

We denote $\mu_{\theta_{0}}=\mu_{\theta_{0}}(t):=\int\left\{H_{0 x}, H_{0 y}\right\}\left(x_{0}(t), y_{0}(t), t+\theta_{0}\right) d t$ and $\kappa_{\theta_{0}}=\kappa_{\theta_{0}}(t)=$ $\int\left(\frac{H_{1 y}}{H_{0 y}}-\delta\left\{H_{0}, H_{1}\right\}\right)\left(x_{0}(t), y_{0}(t), t+\theta_{0}\right) d t$. Then we observe that the Poincaré-ArnoldMelnikov integral $\mu\left(\theta_{0}\right)$, as defined in section 2 , is given by the monodromy of the function $\mu_{\theta_{0}}$ around the closed loop $\gamma$, i.e., the difference between two determinations of the function $\mu_{\theta_{0}}$.

The Picard-Vessiot extension given by the above quadratures is

$$
L_{1} \subset L_{1}\left(\mu_{\theta_{0}}\right):=L_{2} \subset L_{2}\left(\kappa_{\theta_{0}}\right)=L_{\theta_{0}} .
$$

The Galois group $\operatorname{Gal}\left(L_{2} / L_{1}\right)$ is non-trivial if and only if $\mu_{\theta_{0}} \notin L_{1}$ and then $\operatorname{Gal}\left(L_{2} / L_{1}\right)$ $=G_{a}=(\mathbf{C},+)$.

Now we need an elementary result about unipotent groups of complex matrices in dimension 3 which we state as a lemma for future reference.

LEMMA 1. Let

$$
G=\left\{\left(\begin{array}{ccc}
1 & \lambda & \alpha \\
0 & 1 & \mu \\
0 & 0 & 1
\end{array}\right): \lambda \in \mathbf{C}, p_{j}(\lambda, \mu, \alpha)=0, \text { for some } p_{j} \in \mathbf{C}[X, Y, Z]\right\}
$$

be an algebraic subgroup of the group $U_{3}$ of upper triangular complex unipotent $3 \times 3$ matrices. Let $G_{1}$ be the quotient group of $G$ given by the classes of matrices

$$
G_{1}=\left\{\left(\begin{array}{ccc}
1 & \lambda & * \\
0 & 1 & \mu \\
0 & 0 & 1
\end{array}\right)\right\}
$$

Then the following conditions are equivalent:

a) $G$ is not abelian.

b) $\lambda$ and $\mu$ are algebraically independent.

c) $\operatorname{dim} G_{1}=2$.

Proof. As usual, we denote by $G_{a}$ the additive group $(\mathbf{C},+)$. We define the morphism of algebraic groups

$$
\psi: G \rightarrow G_{a}^{2}, \quad\left(\begin{array}{ccc}
1 & \lambda & \alpha \\
0 & 1 & \mu \\
0 & 0 & 1
\end{array}\right) \mapsto(\lambda, \mu)
$$

We observe that this morphism is well-defined because it is given by the restriction to $G$ of the corresponding morphism on $U_{3}$. If $\mu$ depends on $\lambda, \mu=\mu(\lambda)$, then $\mu(\lambda)=a \lambda$, for a fixed $a \in \mathbf{C}$, because $\mu\left(\lambda_{1}+\lambda_{2}\right)=\mu\left(\lambda_{1}\right)+\mu\left(\lambda_{2}\right)$. In this case the group $G$ is abelian. 
Conversely, if $\mu$ and $\lambda$ are independent then we can find matrices

$$
g=\left(\begin{array}{ccc}
1 & \lambda & \alpha \\
0 & 1 & \mu \\
0 & 0 & 1
\end{array}\right), \quad g^{\prime}=\left(\begin{array}{ccc}
1 & \lambda^{\prime} & \alpha^{\prime} \\
0 & 1 & \mu^{\prime} \\
0 & 0 & 1
\end{array}\right)
$$

in $G$ such that $\lambda \mu^{\prime} \neq \lambda^{\prime} \mu$, i.e., the matrices $g$ and $g^{\prime}$ do not commute. We have proved that a) and b) are equivalent.

The equivalence of b) and c) follows easily from the isomorphism $G_{1}=G /$ ker $\psi \approx$ $\psi(G)$, because the algebraic independence of $\lambda$ and $\mu$ is equivalent to $\psi(G)=G_{a}^{2}$.

We remark that from the proof of Lemma 1 it also follows that if any of the conditions a)-c) is satisfied then $G=U_{3}$, because if $\operatorname{ker} \psi$ is trivial then $G \approx G_{a}^{2}$, in contradiction with the fact that $G$ is non-abelian.

Now we apply the above lemma to equation (7). In the representation given by (9), the Galois group of (7) is given by a group $\operatorname{Gal}(P V E)=G$ and $G_{1}=\operatorname{Gal}\left(L_{2} / K\right)=$ $\operatorname{Gal}(P V E) / G a l\left(L_{\theta_{0}} / L_{1}\right)$, with the same notations of Lemma 1. Then $L_{1}=L_{2}$ (i.e., $\left.\mu_{\theta_{0}} \in L_{1}\right)$ if and only if the Galois group $\operatorname{Gal}(P V E)$ of equation (7) is abelian, because the dimension of the group $G_{1}$ is equal to the degree of transcendence of the purely transcendental extension $L_{2} / L_{1}$; and this degree is 1 or 2 , if and only if, $L_{2}=L_{1}$ or $L_{2} \neq L_{1}$, respectively.

The connection with the Poincaré-Arnold-Melnikov integral along a closed loop as studied by Ziglin, formula (6), follows from a simple remark: for any fixed $\theta_{0}$, a necessary condition for $\mu_{\theta_{0}} \in L_{1} \subset \mathcal{M}(\mathbf{C})$ (i.e., for $\operatorname{Gal}(P V E)$ to be abelian) is that the function $\mu_{\theta_{0}}$ must be uniform in $t \in \mathbf{C}$. So the function $\mu\left(\theta_{0}\right)$ must be identically zero.

Problem. Is the above necessary condition a sufficient condition for $\operatorname{Gal}(P V E)$ to be abelian? If the answer to this question is positive, then the function $\mu\left(\theta_{0}\right)$ gives a characterization for the Galois group of the $(P V E)$ to be abelian.

We have proved the following theorem:

Theorem 2. Let $K:=\mathbf{C}\left(e^{2 \pi t / \omega}, e^{i t}\right)$ be the differential field of coefficients of the $(P V E)$ (equation (7)), $\delta:=\int \frac{H_{0} y y}{H_{0 y}^{2}}\left(x_{0}(t), y_{0}(t)\right) d t$ and

$$
L_{1}:=K(\delta), \quad \mu_{\theta_{0}}:=\int\left\{H_{0}, H_{1}\right\}\left(x_{0}(t), y_{0}(t), t+\theta_{0}\right) d t .
$$

Then, under the assumptions (1)-(3) below, a necessary and sufficient condition for the Galois group of the $(P V E)$ to be abelian, for a given $\theta_{0} \in F_{2 \pi}$, is that $\mu_{\theta_{0}} \in L_{1}$. Moreover, a necessary condition for the abelianity of the above Galois group is that the PoincaréArnold-Melnikov integral $\mu\left(\theta_{0}\right)$ must be identically zero over $F_{2 \pi}$.

For the sake of convenience we summarize here the assumptions imposed on system (3) along this section:

Assumption (1). The functions $x_{0}(t), y_{0}(t)$ belong to the field $\mathbf{C}\left(e^{2 \pi t / \omega}\right)$ and the perturbation Hamiltonian $H_{1}$ depends on $x, y$ as a rational function with complex coefficients and on $t$ as a rational function in $e^{i t}$. 
Assumption (2). The general solution of equation (10) is single-valued in $t$, i.e., $\delta \in \mathcal{M}(\mathbf{C})$.

Assumption (3). $\delta \notin K$.

4. Example. The undamped Duffing equation is defined by the Hamiltonian ([7], p. 191)

$$
H=H_{0}+\varepsilon H_{1}=\frac{y^{2}}{2}-\frac{x^{2}}{2}+\frac{x^{4}}{4}-\varepsilon x \cos \theta .
$$

The Hamiltonian $H_{0}$ has the homoclinic orbit $y^{2}=x^{2}-x^{4} / 2$, i.e.,

$$
\Gamma_{0}:\left(x_{0}(t), y_{0}(t)\right)=\left(\frac{\sqrt{2}}{\cosh t},-\frac{\sqrt{2} \sinh t}{\cosh ^{2} t}\right) .
$$

We observe that Assumption (1) is satisfied.

The variational equation of the unperturbed system (10) is given here by

$$
\ddot{\xi}+\left(-1+\frac{6}{\cosh ^{2} t}\right) \xi=0 .
$$

Now we study the Picard-Vessiot extension $K \subset L_{1}$ of (16):

$$
\delta=\int \frac{H_{0 y y}}{H_{0 y}^{2}}\left(x_{0}(t), y_{0}(t)\right) d t=\int \frac{1}{y_{0}(t)^{2}} d t=\frac{\cosh ^{3} t}{4 \sinh t}+\frac{3}{4} t-\frac{3}{4} \cosh t .
$$

As $\delta$ is meromorphic in the complex plane, but $\delta \notin K=\mathbf{C}\left(e^{t}, e^{i t}\right)$, Assumptions (2) and (3) are satisfied and $L_{1}=\mathbf{C}\left(e^{t}, e^{i t}, t\right)$.

The Poincaré-Arnold-Melnikov integral is given here by

$$
\mu\left(\theta_{0}\right)=-\sqrt{2} \int_{\gamma} \frac{\sinh t}{\cosh ^{2} t} \cos \left(\theta_{0}+t\right) d t=2 \sqrt{2} \pi \sin \left(\frac{\pi i}{2}+\theta_{0}\right),
$$

where $\gamma$ is a loop around the pole $t=i \pi / 2$.

As $\mu\left(\theta_{0}\right)$ is not identically zero, the Galois group of the perturbed variational equation of this system is not abelian.

We remark that $\mu\left(\theta_{0}\right)$ has simple zeros and by Theorem 1 , the perturbed separatrix self-intersects transversally.

\section{Final remarks}

5.1. The motivation for Lemma 1 comes from some discussions with Bertrand. In the paper [3] he studies the radical unipotent of the Galois group of linear differential operators given as products of $t$ completely reducible operators. A generalization of Lemma 1 for arbitrary $t$ is implicit in this reference (see Lemma 1.1 and the proof of Theorem 3.2 in [3]) and, for a special case with $t=3$, a criterion of abelianity is explicitly given in Theorem 3 of this reference (our Lemma 1 corresponds to cases i) and ii) of Theorem 3 in [3]). See also [4].

5.2. We remark the possibility of generalizing our approach to the Poincaré-ArnoldMelnikov integral to more degrees of freedom using some results of Goriely and Tabor [6]. These authors studied the relation between the splitting of real separatrices of the 
perturbed system, given by the usual real Poincaré-Arnold-Melnikov integral along a homoclinic orbit, and the branching of solutions in complex time.

5.3. It is clear that in the context of Theorem 2 , if the function $\mu\left(\theta_{0}\right)$ has simple zeros, then it is not identically zero. Hence, the condition for non-abelianity is stronger that the condition for the self-intersection of separatrices as given by Ziglin in Theorem 1. However, if the function $\mu\left(\theta_{0}\right)$ has simple zeros, then the unperturbed system is not integrable by holomorphic first integrals. We conjectured that the more general condition of non-abelianity of the Galois group of (7) is also a sufficient condition for the nonintegrability of system (3).

The above conjecture should be considered in the context of some kind of generalization of the following joint result of Ramis and the author.

Consider a complex analytic symplectic manifold $M$ of dimension $2 n$ and let $X_{H}$ be an analytic Hamiltonian system defined on $M$. Let $\Gamma$ be the Riemann surface corresponding to an integral curve $C$ defined by $z=z(t)$ (which is not an equilibrium point) of $X_{H}$. Then we can obtain the variational equation $(V E)$

$$
\dot{\eta}=X_{H}^{\prime}(z(t)) \eta
$$

along $\Gamma$. The coefficient field of this system is defined as the field of meromorphic functions $\mathcal{M}(\bar{\Gamma})$ over some completion $\bar{\Gamma}$ of $\Gamma$ : essentially we add the singular points and (perhaps) points at the infinity (for more details see [13] or [12]).

Then, in the above situation, we have proved in [13] the following result:

THEOREM 3. Assume that there are $n$ meromorphic first integrals of $X_{H}$ in involution and independent in a neighborhood of the curve $C$ in $M$, not necessarily on $C$ itself. Then the identity component $G^{0}$ of the Galois group $G$ of the (VE) is an abelian subgroup of the symplectic group.

We observe that up to now in all the applications of the above theorem only the normal variational equation $(N V E)$ is used. The $(N V E)$ is obtained from the $(V E)$ by a reduction process ([13] and [12]). Here, we have to consider the complete $(V E)$. Furthermore, in the proof of the above theorem, it was proved that when the identity component of the Galois group of $(V E)$ along $\Gamma$ is not abelian then the Hamiltonian system has not a complete set of meromorphic integrals in involution a neighborhood of $\Gamma$.

The (possible) connection of Theorem 3 with the Poincaré-Arnold-Melnikov method is as follows. First, we observe that the Galois group of equation (7) is connected, i.e., $G=$ $G^{0}$. Now we can embed system (3) in an autonomous 3 -degrees of freedom Hamiltonian system $X_{\bar{H}}$, by considering the energy $e$ and the parameter $\varepsilon$ as new variables (they are two first integrals in involution of this new system), i.e., $\bar{H}(x, y, \theta, e, \varepsilon, p):=H(x, y, \theta, \epsilon)-$ $e$, where $p$ is the canonical conjugate variable of $\varepsilon$ (a cyclic variable). This system is very degenerate: it splits in a two degrees of freedom Hamiltonian system which contains system (3) and a trivial 1-degree of freedom Hamiltonian system in the variables $(\varepsilon, p)$. Then the $P V E$ is contained in the $V E$ along the integral curve (5).

If we apply Theorem 3 to the Hamiltonian $\bar{H}(x, y, \theta, e, \varepsilon, p)$ along the integral curve (5), in general, we do not obtain a non-integrability criterion as in Theorem 1 . This is due to the fact that, for general systems, the Riemann surface associated to $\Gamma_{\theta_{0}}$, for a 
fixed $\theta_{0}$, is the complex plane. Then the coefficient field is $\mathcal{M}\left(\bar{\Gamma}_{\theta_{0}}\right)=\mathcal{M}(\mathbf{C})$, and the only contribution to the Galois group comes from the monodromy given by the PoincaréArnold-Melnikov integral. In other words, with the notation of section $3, L_{1}$ is contained in the coefficient field of the variational equation. This happens, for instance, in the example of section 4 . In order to bring Theorem 1 into this picture, it seems necessary to generalize Theorem 3 to larger dynamical objects than integral curves, i.e., to singular Lagrangian manifolds.

For some particular systems it is possible to prove the equivalence of Theorems 2 and 3 (and, hence, the link between Theorems 1 and 3). For instance, in the case when the perturbation $H_{1}$ depends on time as an elliptic function with real period $2 \pi$ and with the same imaginary period $i \omega$ as the unperturbed separatrix $\Gamma_{0}$. Then the Riemann surface of the integral curve is given by the cylinder $F_{i \omega}$, and the monodromy group of the variational equation is generated by two kinds of monodromies: one of them given by the monodromy along the imaginary period and the other one by the Poincaré-ArnoldMelnikov integral $\mu\left(\theta_{0}\right)$. For example, the Hamiltonian system defined by $H=\frac{1}{2} y^{2}-$ $\frac{2 \pi^{2}}{\omega^{2}} x^{2}+\frac{1}{2} x^{3}+\varepsilon y \frac{\wp \prime(t)}{\wp(t)}$, where $\wp$ is the Weierstrass elliptic function of periods $2 \pi$ and $i \omega$, satisfy this condition; we leave the details to the reader.

Acknowledgements. The author is indebted to Daniel Bertrand, Josep-Maria Peris, Jean-Pierre Ramis and Carles Simó for their interest in this paper and for interesting discussions. This work was partially supported by the Spanish grant DGICYT PFM20000805 and the Catalan grant CIRIT 2001SGR-70.

\section{References}

[1] V. I. Arnold, Instability of dynamical systems with several degrees of freedom, Dokl. Akad. Nauk SSSR 156 (1964), 581-585 (in Russian).

[2] V. I. Arnold, Équations différentielles ordinaires, Mir, Moscou, 1974.

[3] D. Bertrand, Unipotent radicals of differential Galois groups, Math. Ann. 321 (2001), 645-666.

[4] D. Bertrand, Extensions panachées et dualité, preprint 2001.

[5] R. C. Churchill, Differential Galois Theory (Class Notes), Department of Mathematics, Graduate Center, City University of New York, Spring 1999.

[6] A. Goriely and M. Tabor, The singularity analysis for nearly integrable systems: homoclinic intersections and local multivaluedness, Physica D 85 (1995) 93-125.

[7] J. Guckenheimer and P. Holmes, Nonlinear Oscillations, Dynamical Systems, and Bifurcations of Vector Fields, Springer-Verlag, New York, 1983.

[8] I. Kaplansky, An Introduction to Differential Algebra, Hermann, Paris, 1976.

[9] A. R. Magid, Lectures on Differential Galois Theory, Amer. Math. Soc., Providence, RI, 1994.

[10] J. Martinet and J. P. Ramis, Théorie de Galois différentielle et resommation, in: Computer Algebra and Differential Equations, E. Tournier (ed.), Academic Press, London, 1989, $117-214$.

[11] V. K. Melnikov, On the stability of the center for time-periodic perturbations, Trans. Moscow Math. Soc. 12 (1963), 3-52. 
[12] J. J. Morales-Ruiz, Differential Galois Theory and Non-Integrability of Hamiltonian Systems, Progr. Math. 179, Birkhäuser, Basel, 1999.

[13] J. J. Morales-Ruiz and J. P. Ramis, Galoisian obstructions to integrability of Hamiltonian systems I, II, Methods Appl. Anal. 8 (2001), 33-95, 97-111.

[14] H. Poincaré, Les méthodes nouvelles de la mécanique céleste, Tome III, Gauthier-Villars, Paris, 1899.

[15] S. L. Ziglin, Self-intersection of the complex separatrices and the non-existence of the integrals in the Hamiltonian systems with one-and-half degrees of freedom, J. Appl. Math. Mech. 45 (1982), 411-413.

[16] S. L. Ziglin, Splitting of separatrices, branching of solutions and nonexistence of an integral in the dynamics of a solid body, Trans. Moscow Math. Soc. 1 (1982), 283-298. 